\title{
Fault Diagnosis of Wind Turbine Blades based on Wavelet Theory and Neural Network
}

\author{
Junxi $\mathrm{Bi}^{\mathrm{a}}{ }^{\mathrm{a}}$, Chenglong Zheng ${ }^{\mathrm{b}, *}$, Hongzhong Huang ${ }^{\mathrm{c}}$, Xiaojuan Song ${ }^{\mathrm{b}}$, and Jinfeng Li ${ }^{\mathrm{d}}$ \\ ${ }^{a}$ Aviation College, Inner Mongolia University of Technology, Hohhot, 010051, China \\ ${ }^{b}$ College of Mechanical Engineering, Inner Mongolia University of Technology, Hohhot, 010051, China \\ 'Institute of Reliability Engineering, University of Electronic Science and Technology of China, Chengdu, 611731, China \\ ${ }^{d}$ Inner Mongolia Institute of Metrology Testing and Research, Hohhot, 010020, China
}

\begin{abstract}
With the development of the wind turbine industry, the reliability requirements of wind turbine blades are continuously increasing. In this paper, static load fatigue experiments are carried out on wind turbine blades, and the collected fault data of blades are extracted using the wavelet transform method. Wavelet theory is applied to remove the noise of the data and eliminate the interference of noise on the fault diagnosis of wind turbine blades. Then, the wavelet decomposition method is used to decompose high frequency signals and low frequency signals. The faulty low frequency signals are extracted and analyzed in the time domain, and a fault diagnosis method of wind turbine blade is established. The data of different vibration frequencies of wind turbine blades are collected by the acquisition system, and the data are imported into the neural network. The neural network is used to process the data and identify the states of wind turbine blades. The neural network proves that the wavelet transform method has reliable fault diagnosis ability in time domain analysis.
\end{abstract}

Keywords: fatigue experiment; fault data; wavelet transform method; time domain analysis; fault diagnosis; neural network

(Submitted on April 13, 2019; Revised on May 25, 2019; Accepted on June 25, 2019)

(C) 2019 Totem Publisher, Inc. All rights reserved.

\section{Introduction}

With the depletion of traditional energy sources, new energy sources have attracted worldwide attention. It is necessary to solve the problem of energy shortages, and more attention has been paid to the development and utilization of renewable energy. A form of renewable energy, wind energy does not cause pollution to the environment and is cheap and easy to obtain. Wind power enterprises are paying more attention to the reliable and stable operation of wind turbine equipment. Because the wind turbine blade is a crucial component of wind turbine equipment, the reliable operation of wind turbines cannot be separated from the reliable operation of wind turbine blades. Therefore, it is meaningful to analyze the decomposed signals of different stages by using signal processing software. It is necessary to monitor and deal with some faults of wind turbine blades.

\section{Mathematical Modeling}

In the long running of wind turbine blades, faults often occur in the form of cracks due to various environmental factors [1]. With the continuous propagation of blade cracks, wind turbine blades eventually break and fail. The crack propagation of wind turbine blades is generally divided into three stages: the crack initiation stage, the stable crack propagation stage, and the rapid crack propagation stage. For these three stages, the wavelet transform method is applied to identify the damage signals of the blade, and then the effective fault feature signals are extracted.

The wavelet transform method characterizes the part features of signals in the time-frequency domain and has better time and frequency resolution in various frequency signals [2].

\footnotetext{
* Corresponding author.

E-mail address: 1597123651@qq.com, 574346498@qq.com
} 
The basic wavelet transform formula is obtained as follows:

$$
\psi_{\alpha, \beta}(t)=\frac{1}{\sqrt{\alpha}} \psi\left(\frac{t-\beta}{\alpha}\right)
$$

Here, $\alpha$ is the scale factor, $\beta$ is the shift factor, and $\psi(t)$ is the basic function of the wavelet. $\psi_{\alpha, \beta}(t)$ is a kind of wavelet function obtained by stretching and shifting the basic wavelet function.

The following continuous wavelet transform function is obtained by integral transform:

$$
\psi(\alpha, \beta)=\frac{1}{\sqrt{\alpha}} \int_{-\infty}^{+\infty} f(t) \psi_{\alpha, \beta}(t) \mathrm{d} t
$$

Here, $f(t)$ is an input signal.

When any parameter of the scale factor $\alpha$ and the shift factor $\beta$ changes, a different level of wavelet function can be formed. As the scale factor $\alpha$ increases, the time resolution of the wavelet will decrease and the corresponding frequency resolution will increase. As the scale factor $\alpha$ decreases, the time resolution of the wavelet will increase and the corresponding frequency resolution will decrease. The wavelet transform signals have higher time resolution at the high frequency part and higher frequency resolution at the low frequency part [3]. The shift factor $\alpha$ can determine the shift degree of the wavelet function and form some wavelets with different phases, and it also reflects the signal components of different frequency parts.

When a wind turbine runs, the output signal of the fault blade is composed of deterministic signals and random noise signals. Its wavelet transform is the sum of the corresponding two partial wavelet transforms.

The noise signal is mainly the sudden fluctuation of the signal [4]. It is easy to make wrong judgments for the identification of blade faults. In order to remove the influence of noise signals on the fault identification of wind turbine blades, it is necessary to remove the noise signal by wavelet transform according to the different features of the Lipschitz exponent of the noise signal and fault signal.

The Lipschitz exponent is extended to measure the singularity of the function in $0 \leq \lambda<1$.

When $0 \leq \lambda<1$, for all $t_{0} \in[a, b]$, the singularity of the signal is expressed as follows:

$$
\left|f(t)-f\left(t_{0}\right)\right| \leq A\left|t-t_{0}\right|^{\lambda}
$$

Here, $A$ is the singular influence factor.

The function $f(t)$ is continuous on the interval $[a, b]$, and the condition of Lipschitz exponent $\lambda$ is obtained as follows:

$$
|D W T(\beta)| \leq B\left(2^{k}\right)^{\lambda}
$$

Here, $k$ is the scaling factor of the discrete wavelet transform.

Logarithms are taken on both sides of the above formula to obtain the following formula.

$$
\log _{2}|D W T(\beta)| \leq \log _{2} B+k \lambda
$$

Here, $\log _{2}|D W T(\beta)|$ is the wavelet transform coefficient.

The Lipschitz exponent $\lambda$ of a continuous signal is positive, and the wavelet transform coefficient $k$ increases with an increase in the scale factor of discrete wavelet transform. The Lipschitz exponent $\lambda$ of a white noise signal is negative, and 
the wavelet transform coefficient $k$ decreases with an increase in the scale factor of discrete wavelet transform [5]. According to the difference of wavelet transform coefficients of continuous signals and noise signals, the different propagation of signal can be found [6]. The noise signal in the fault signals can be removed by this method.

Irregular mutations and singularities in signals usually contain important information, and they are effective feature information of fault signals [7]. The singularity of the signal is that the amplitude or frequency of the signal changes suddenly at a certain moment.

The relationship between the modulus maximum of wavelet transform and the singularities of signals is expressed as follows:

If $h(t)$ is a smooth function and satisfies the conditions $\int_{-\infty}^{+\infty} h(t) \mathrm{d} t=1$ and $\lim _{t \rightarrow 0} h(t)=0$, the formula can be obtained as follows:

$$
h(t)=\frac{1}{\sqrt{2 \pi} \sigma} e^{-\frac{t^{2}}{2 \sigma}}
$$

Here, $\sigma$ is the distribution coefficient of the Gaussian function.

When $\psi(t)=\mathrm{d} h(t) / \mathrm{d} t, \int_{-\infty}^{+\infty} \psi(t) \mathrm{d} t=0$. Therefore, the function $\psi(t)$ can be selected as the wavelet basis function.

The wavelet transform formula for the input function [8] $f(t)$ with respect to function $\psi(t)$ is obtained as follows:

$$
\psi_{f}(\alpha, \beta)=\frac{1}{\sqrt{|\alpha|}} \int_{-\infty}^{+\infty} f(t) \psi\left(\frac{t-\beta}{\alpha}\right) \mathrm{d} t
$$

Here, $\psi_{f}(\alpha, \beta)$ can also be written as the following expression:

$$
\psi_{f}(\alpha, \beta)=-|\alpha| \frac{\mathrm{d}}{\mathrm{d} \beta} \int_{-\infty}^{+\infty} f(t) g_{\alpha \beta}(t) \mathrm{d} t
$$

Here, $g_{\alpha \beta}(t)=\left(\frac{1}{\sqrt{|\alpha|}}\right) g\left(\frac{t-\beta}{\alpha}\right)$ is the Gaussian function.

When $\alpha>0$, the following formula can be obtained from Equation (8):

$$
\left|\psi_{f}(\alpha, \beta)\right|=\alpha\left|\frac{\mathrm{d}}{\mathrm{d} \beta} \int_{-\infty}^{+\infty} f(t) g_{\alpha \beta}(t) \mathrm{d} t\right|
$$

$\int_{-\infty}^{+\infty} f(t) g_{\alpha \beta}(t) \mathrm{d} t$ is the integral formula of input signal function $f(t)$.

When $\alpha$ is very small, $g_{\alpha \beta}(t)$ has little influence on the mutation part of wavelet transform $f(t)$. The modulus of wavelet transform $\left|\psi_{f}(\alpha, \beta)\right|$ is proportional to the first derivative of $\int_{-\infty}^{+\infty} f(t) g_{\alpha \beta}(t) \mathrm{d} t$ with the scale factor $\alpha$ as the coefficient. Therefore, the maximum value of modulus $\left|\psi_{f}(\alpha, \beta)\right|$ after wavelet transform corresponds to the mutation point of $\int_{-\infty}^{+\infty} f(t) g_{\alpha \beta}(t) \mathrm{d} t$. When $\alpha$ is very small, the mutation point of $\int_{-\infty}^{+\infty} f(t) g_{\alpha \beta}(t) \mathrm{d} t$ can be approximately regarded as the mutation point of the input signal $f(t)$. 
When $\alpha$ is very large, the attenuation of $\psi_{f}(\alpha, \beta)$ is exponential as the scale factor $\alpha$ increases. The larger the Lipschitz exponent, the faster the attenuation.

Because there is a certain correspondence between the modulus maximum point and the signal mutation point, the mutation information of the signal is characterized in the high frequency part of the wavelet transform. Therefore, the modulus maximum point of the wavelet transform can be analyzed to determine the feature information of the signal mutation point.

The acquired signals are processed by wavelet transform, and the singular relation between the modulus maximum of the processed signal and the fault signal is found [9]. The singularity points after wavelet transform are determined, and then the fault location of wind turbine blade can be found by using the singularity points.

\section{Experimental Data Acquisition and Algorithm Application}

In this experiment, an experiment scheme of single blade static load is adopted [10]. Many internal and external factors need to be considered when the airfoil blade is chosen for the experiment.

Table 1 shows some types of airfoil blades and the classification of different airfoil blades.

Table 1. NACA series parameters of blade airfoil

\begin{tabular}{|c|c|c|c|c|c|}
\hline Airfoil & Cross section & Radium (m) & Inflow angle & Installation angle $\left(^{\circ}\right)$ & Angle of attack $\left(^{\circ}\right)$ \\
\hline NACA4418 & $\mathrm{A}-\mathrm{A}$ & 1.5 & 50.63 & 45.23 & 5.75 \\
\hline NACA4418 & B-B & 3 & 35.41 & 30.34 & 5.75 \\
\hline NACA4418 & $\mathrm{C}-\mathrm{C}$ & 4.5 & 31.23 & 25.16 & 5.75 \\
\hline NACA4418 & D-D & 6 & 24.32 & 20.19 & 5.75 \\
\hline NACA4418 & E-E & 8 & 21.36 & 17.51 & 5.75 \\
\hline NACA4418 & F-F & 10.5 & 17.15 & 14.02 & 5.75 \\
\hline NACA4418 & G-G & 13 & 15.43 & 12.35 & 5.75 \\
\hline NACA4418 & $\mathrm{H}-\mathrm{H}$ & 14.5 & 14.67 & 10.76 & 5.75 \\
\hline NACA4415 & I-I & 16 & 13.90 & 9.54 & 5.5 \\
\hline NACA4415 & $\mathrm{J}-\mathrm{J}$ & 18 & 11.45 & 8.21 & 5.5 \\
\hline NACA4415 & K-K & 20 & 10.13 & 7.06 & 5.5 \\
\hline NACA4415 & L-L & 21.5 & 9.42 & 6.32 & 5.5 \\
\hline NACA4415 & M-M & 23 & 8.76 & 5.74 & 5.5 \\
\hline NACA4415 & $\mathrm{N}-\mathrm{N}$ & 24 & 8.46 & 4.23 & 5.5 \\
\hline NACA4415 & $\mathrm{O}-\mathrm{O}$ & 25 & 7.98 & 3.89 & 5.5 \\
\hline NACA4415 & P-P & 26.5 & 7.65 & 3.15 & 5.5 \\
\hline NACA4415 & Q-Q & 27.5 & 7.52 & 2.87 & 5.5 \\
\hline NACA4412 & R-R & 29 & 6.93 & 2.43 & 5.25 \\
\hline NACA4412 & S-S & 30.5 & 6.62 & 2.12 & 5.25 \\
\hline NACA4412 & $\mathrm{T}-\mathrm{T}$ & 32 & 6.41 & 1.91 & 5.25 \\
\hline NACA4412 & U-U & 34 & 6.18 & 1.64 & 5.25 \\
\hline NACA4412 & $\mathrm{V}-\mathrm{V}$ & 35.5 & 5.94 & 1.38 & 5.25 \\
\hline NACA4412 & $\mathrm{W}-\mathrm{W}$ & 37 & 5.73 & 1.14 & 5.25 \\
\hline NACA4412 & $\mathrm{X}-\mathrm{X}$ & 38 & 5.58 & 0.82 & 5.25 \\
\hline
\end{tabular}

Due to the limited experimental conditions, a $1.5 \mathrm{~m}$ NACA4418 airfoil blade is selected in the fatigue test. Its material is glass fiber, the average width of the test blade is $130 \mathrm{~mm}$, and the thickness of the blade skin is $4 \mathrm{~mm}$.

The specific materials of wind turbine blade are shown in Table 2.

Table 2. Wind turbine blade material table

\begin{tabular}{|c|c|c|c|c|}
\hline Resin content & Resin density & Fiber content $\left(\mathrm{g} / \mathrm{cm}^{2}\right)$ & Fiber density $\left(\mathrm{g} / \mathrm{cm}^{2}\right)$ & Fiber volume ratio \\
\hline $35 \%$ & 1.1 & $65 \%$ & 2.7 & $50 \%$ \\
\hline
\end{tabular}

It is difficult to monitor the entire process of crack propagation, but it can be simulated by prefabricated short cracks [11]. The blade needs to be seen as a cantilever beam, and the blade root is fixed by the flange. Cyclic loads are imposed to the tip of the blade to make the blade continuously vibrate, so the vibration state of the wind turbine blade is simulated under the actual wind load [12].

After comprehensive consideration of various factors, the NACA4418 airfoil blade is selected for the fatigue experiment [13]. Crack prefabrication is carried out on the blade, and the prefabricated crack is at a certain position in the 
middle of the blade. A small saw is used to cut a transverse notch that is $0.2 \mathrm{~mm}$ deep, $2 \mathrm{~cm}$ long, and $0.5 \mathrm{~mm}$ wide.

An acoustic emission acquisition system of two-channel PXWAE [14] is used in the experiment, and the frequency range is $3 \mathrm{kHz}$ to $300 \mathrm{kHz}$. The acquisition part is composed of an AE sensor, preliminary amplifier, vibration source, NI information acquisition card, and computer.

The following Figure 1 is a simplified diagram of the blade fatigue experiment.

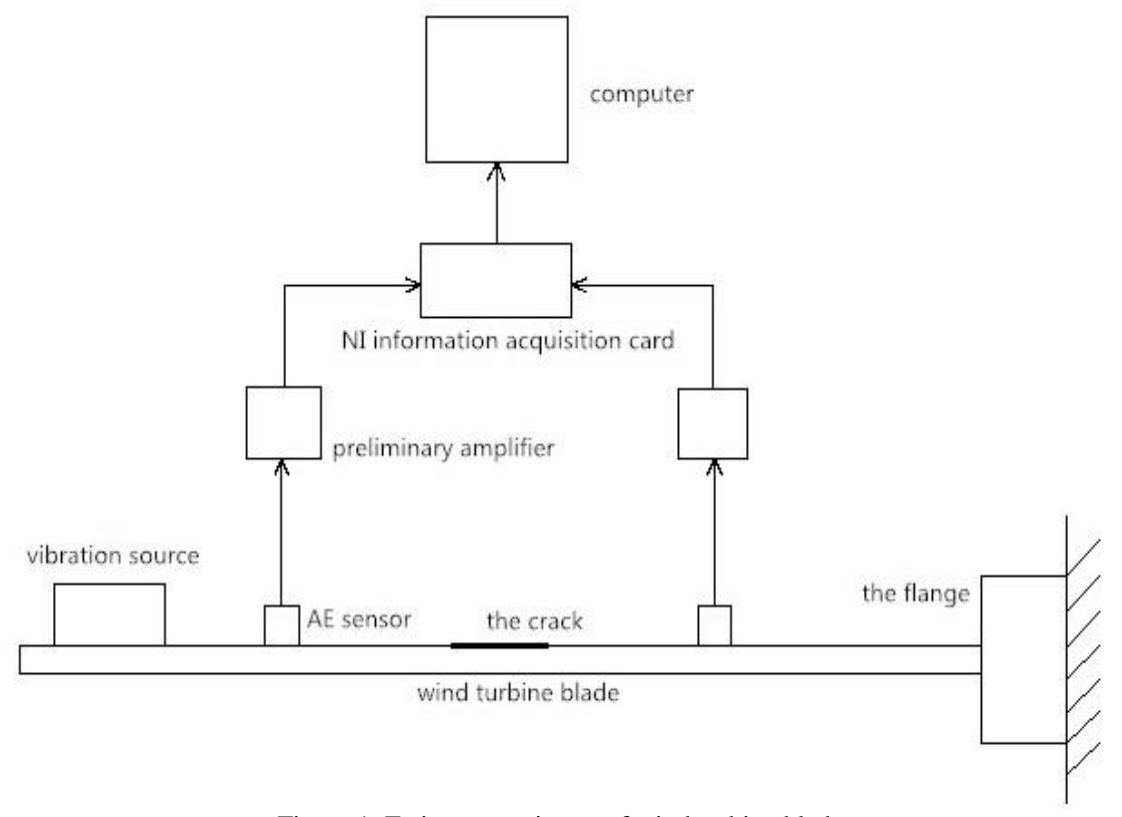

Figure 1. Fatigue test picture of wind turbine blade

The collected signals are analyzed using the wavelet transform method on the LabVIEW software platform. The noise interference signal is removed, and then the signal is processed by wavelet decomposition [15].

Figure 2 shows the original data collected by the aforementioned acoustic emission system, and the signal is a relatively standard periodic signal [16]. It can be seen that the collected data is a continuous signal that reflects a large amount of information in a small time interval.

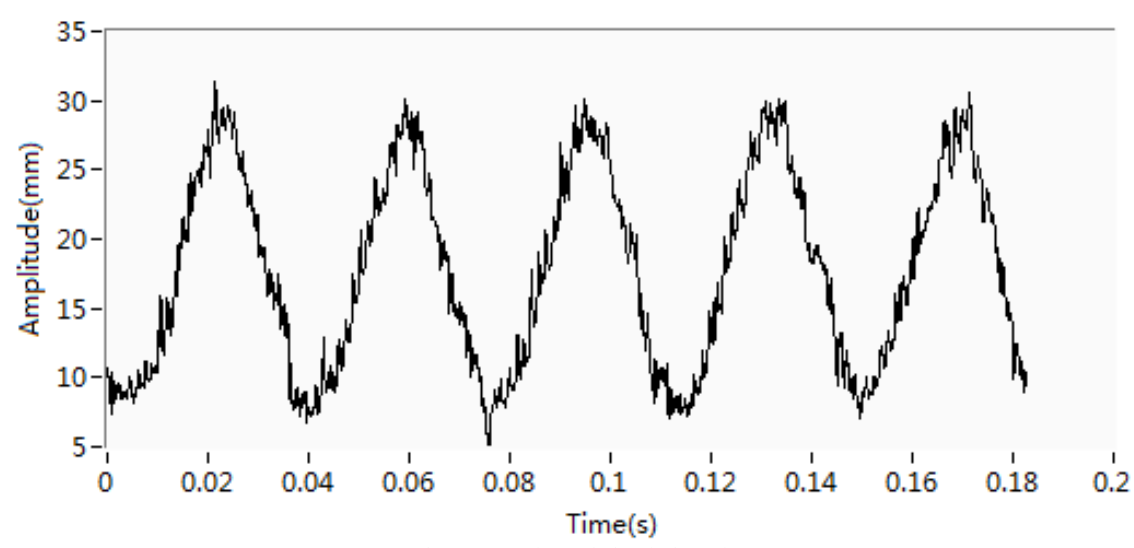

Figure 2. The original signal

The accuracy of data reaches $0.01 \mathrm{~s}$, which is quite accurate in the data collection of the fault blade.

The original signal above cannot directly reflect the running states of wind turbine blades, which requires signal processing [17]. The signal is stored on the computer in the form of original data, and then it is imported into the data processing software LabVIEW. The wavelet transform method is used to process and analyze signals in the LabVIEW software. 
As can be seen in Figure 2, the original signal mixes the higher frequency noise signal besides the effective signal. In order to further extract the effective signals of wind turbine blades, it is necessary to filter the original signal.

Firstly, the original signal is processed by wavelet discrete transform. Then, the coif5 wavelet is selected as a discrete signal, which is divided into six levels.

The following Figure 3 shows that the discrete wavelet signal is divided into six levels.

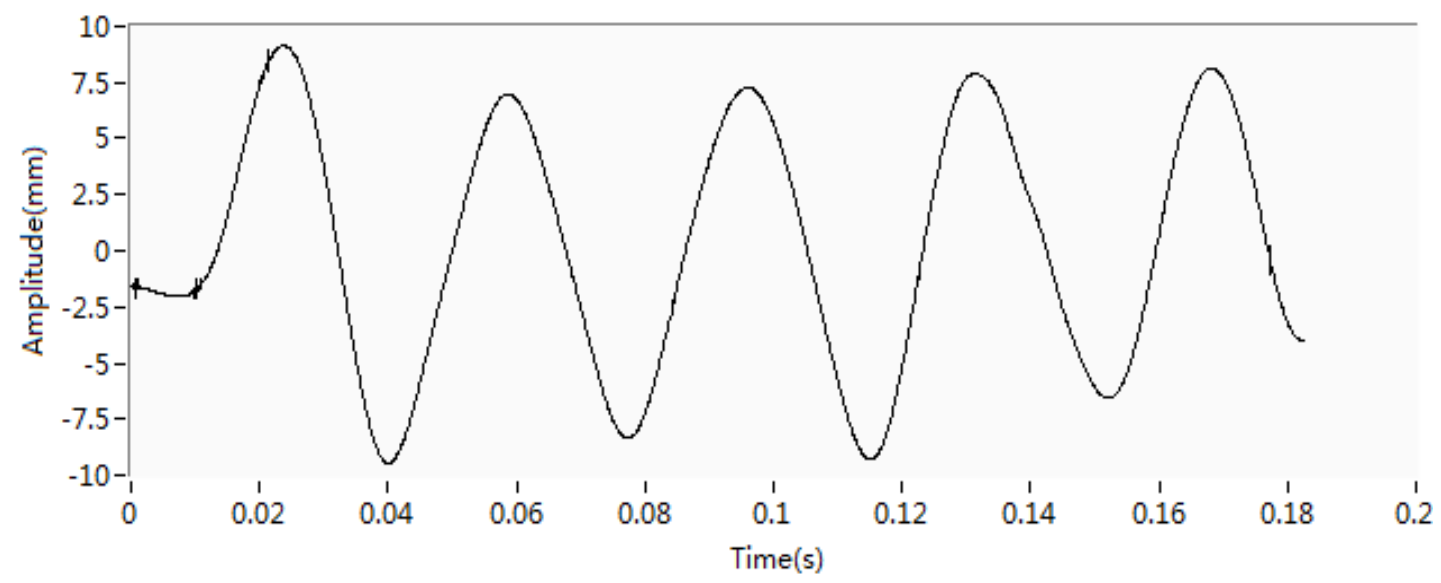

Figure 3. The discrete wavelet signal

The effective signal in the original signal has a relatively large vibration value and a relatively large period [18]. The noise signal has a relatively small amplitude and a relatively small period. The high frequency noise signal in the original signal is removed by discrete wavelet transform, and the effective fault signal of the blade is successfully retained.

In Figure 3, it can be seen that the noise-reduced signal is relatively smooth. After the noise is reduced in the original signal, the system will speed up the operation of the signal in subsequent operations. At the same time, it is easier to find the singular mutation point of the fault signal, and the fault recognition ability of the blade will increase. The original signal is processed using the discrete wavelet method to reduce the noise of the external environment and the internal system, which makes it easier to extract fault signals.

The signal in Figure 3 is amplified to obtain the signal in Figure 4. The amplified signal has a certain increase in the time domain, and the corresponding signal period will also increase. The signal is more intuitive, and the mutation value of the discrete amplified signal can be clearly seen [19]. The feature information of the signal can be easily obtained in Figure 4.

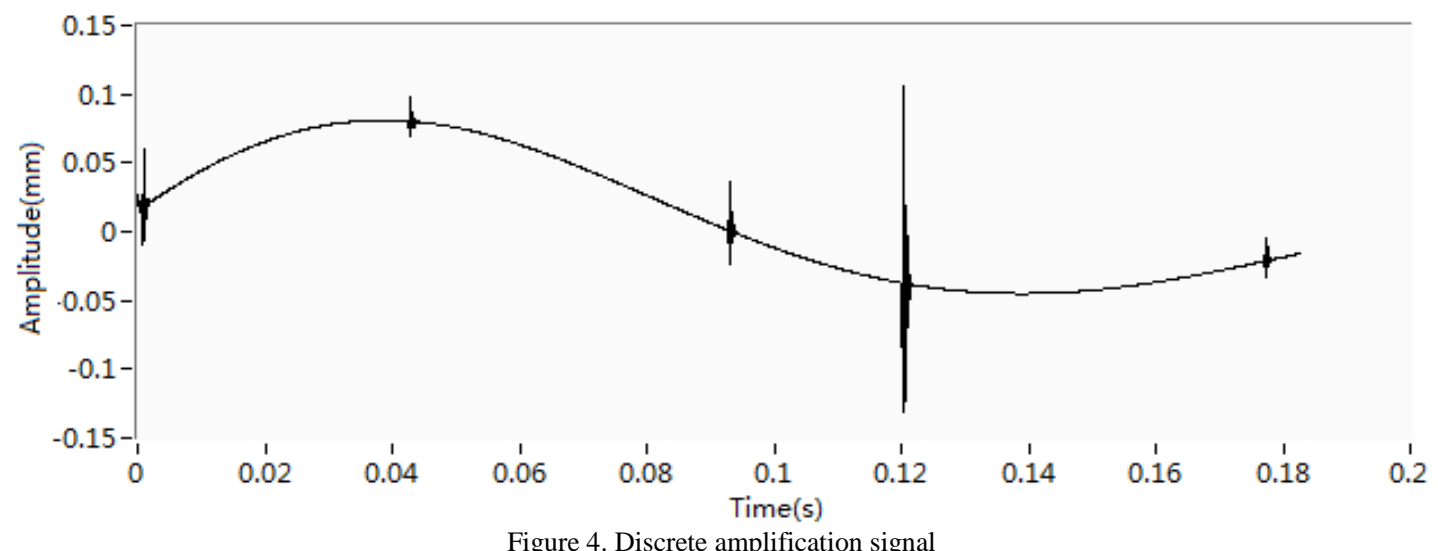

The discrete wavelet signal in Figure 4 is decomposed to obtain the fault feature signal in the effective signal. The wavelet decomposition method is needed for feature extraction of effective signals [20].

The wavelet decomposition tree is shown in Figure 5. 


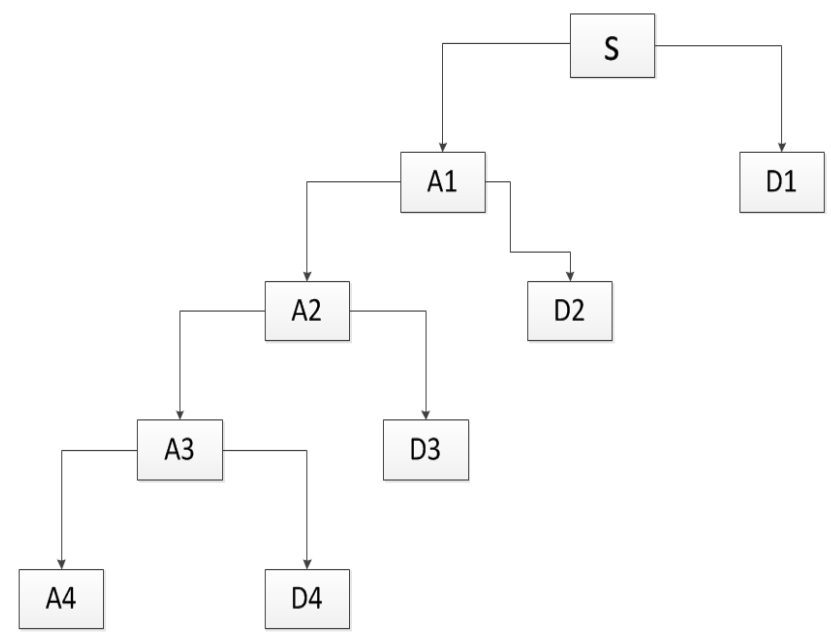

Figure 5. The wavelet decomposition tree structure

The noise-reduced signal is decomposed by wavelet transform in Figure 5. The signal S represents the noise-reduced signal. According to the different frequencies of the signal, the noise-reduced signal is decomposed into four layers. The high frequency part of the signal is separated, and the low frequency signal is decomposed layer by layer. The high frequency signal in the lower frequency part is obtained, and the hidden fault signal of the wind turbine blade can be found [20]. In order to further study the fault feature signals of wind turbine blades, some important feature information must be obtained by this method. The extracted fault feature signals are used to identify the running states of wind turbine blades.

The above discrete wavelet signal is decomposed into four layers, and the specific decomposition results are shown in Figure 6.

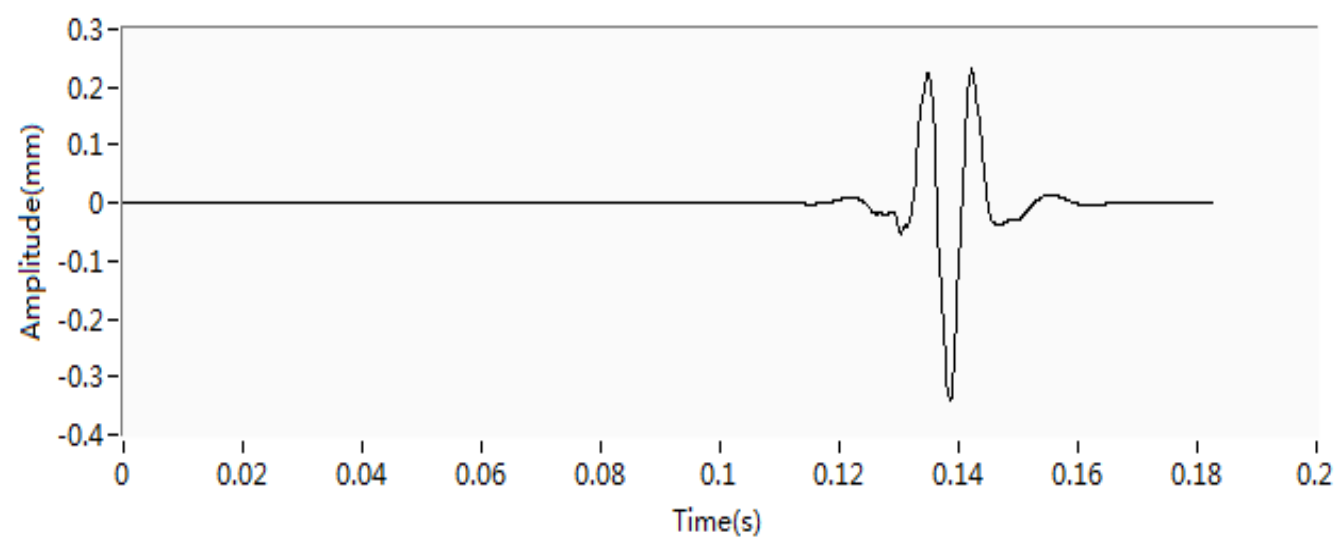

Figure 6. The second layer decomposition signal

The signal $\mathrm{S}$ is decomposed in the second layer to obtain the signal, as shown in Figure 6. Figure 6 is an ideal fault signal of wind turbine blades. In this figure, amplitude peaks occur at about $0.14 \mathrm{~s}$. According to the singular mutation information corresponding to the modulus maximum part of the fault, it can be judged that this is an important feature information part in the fault signal of wind turbine blades.

Some important features of the next layer signal are contained in the second layer decomposition signal, which can predict some fault features of the subsequent signals.

Because the fault information extracted from the third layer is extremely similar to the fault features of the second layer, it is not further explained and analyzed here. The second layer is decomposed to obtain a low frequency signal of the third layer.

The low frequency signal of the third layer is decomposed in the fourth layer [21], and the signal figure of the fourth layer is obtained in Figure 7. 


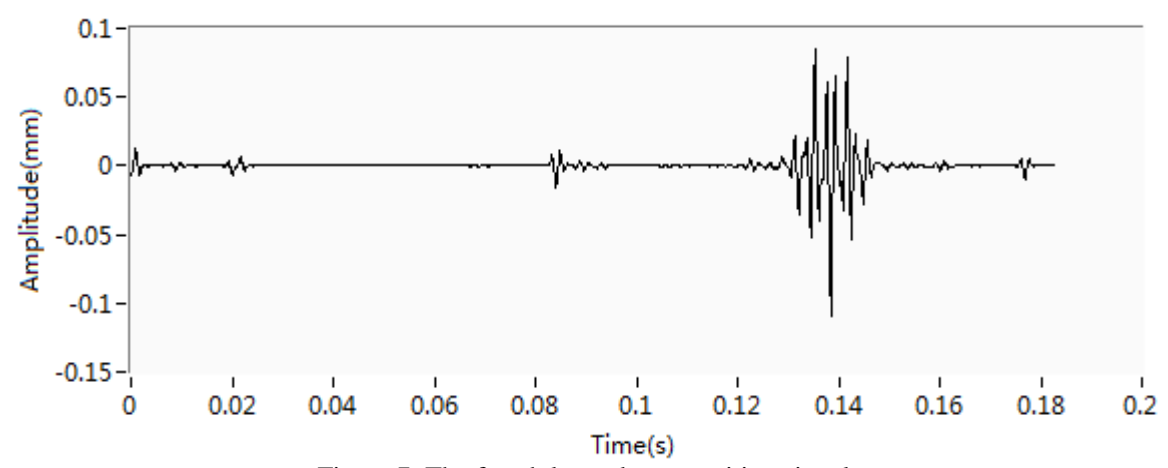

Figure 7. The fourth layer decomposition signal

In the decomposition signal of the fourth layer, some mutation parts can be found. The maximum amplitude of the mutation part also occurs at about $0.14 \mathrm{~s}$, which is extremely consistent with the fault location of the second layer decomposition signal. Through the two-layer data, it is also verified that the determination of wind turbine blade faults is correct.

The following Figure 8 is a wavelet decomposition figure of the fault signal in discrete amplified signals.

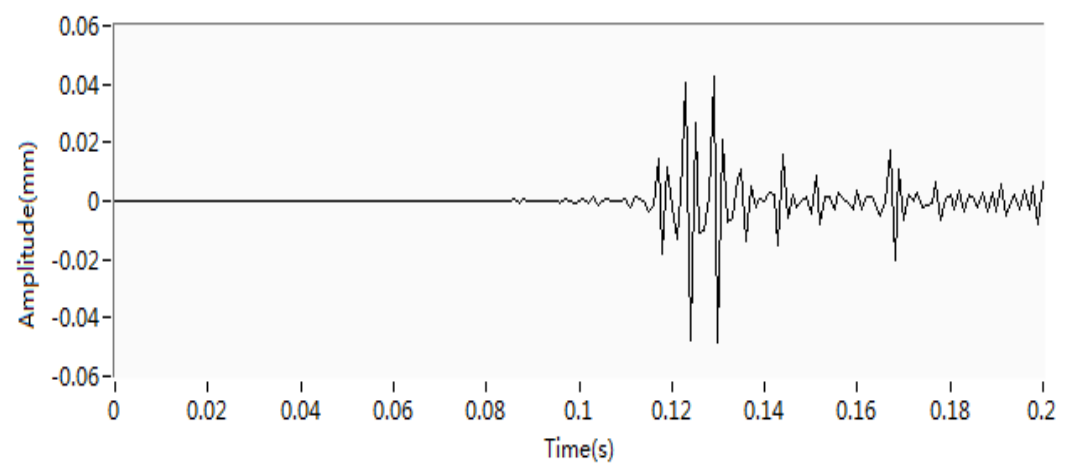

Figure 8. The attenuation curve of fault signal

The fault feather signal of wind turbine blades shows the form of exponential attenuation in the time domain [22], and it has a short duration. The attenuation feather of the fault signal can be utilized to find the fault signal of the blade.

The vibration signals of wind turbine blades have different frequency bands [23]. The vibration signal is divided into six frequency bands: (0.00-0.39) $f,(0.40-0.79) f,(0.80-0.99) f, f,(2-3) f$, and $\geq 4 f$. Then, the six bands of the signal are used as input data of the neural network. The blade state is divided into four states: normal state, crack state, wear state, and icing state.

The input data of the neural network is shown in Table 3.

Table 3. Input samples of the neural network

\begin{tabular}{|c|c|c|c|c|c|c|}
\hline Blade state & $(0.00-0.39) f$ & $(0.40-0.79) f$ & $(0.80-0.99) f$ & $f$ & $(2-3) f$ & $\geq 4 f$ \\
\hline Normal & 0.00 & 0.00 & 0.00 & 0.90 & 0.06 & 0.07 \\
\hline Crack & 0.50 & 1.10 & 0.00 & 0.60 & 0.01 \\
\hline Abrasion & 0.00 & 0.20 & 0.30 & 0.01 & 0.70 \\
\hline Icing & 0.00 & 0.40 & 0.20 & 0.50 & 0.07 \\
\hline
\end{tabular}

The output data of the neural network is shown in Table 4.

Table 4. Output samples of the neural network

\begin{tabular}{|c|c|c|c|c|}
\hline Blade state & O1 & O2 & O2 & O4 \\
\hline Normal & 0 & 0 & 0 & 0 \\
\hline Crack & 0 & 1 & 0 & 0 \\
\hline Abrasion & 0 & 0 & 0 & 1 \\
\hline Icing & 0 & 0 & 1 & 0 \\
\hline
\end{tabular}


The data in Tables 3 and 4 are trained by the neural network in Matlab software. In the data test module, there are four input points and four output points of the wind turbine blade, and the hidden calculation layer is 10. In the whole data, the training data is $70 \%$, the verification data is $15 \%$, and the test data is $15 \%$. The training data, verification data, and test data are automatically filtered by the system from the whole data. This avoids the subjective selection of data by humans, and it is beneficial to ensure the reliability of test results. The machine automatically selects the test data more quickly, and the error of data processing is also small [24].

After the data in Tables 3 and 4 are processed by neural network operation, the performance curve can be obtained in Figure 9.

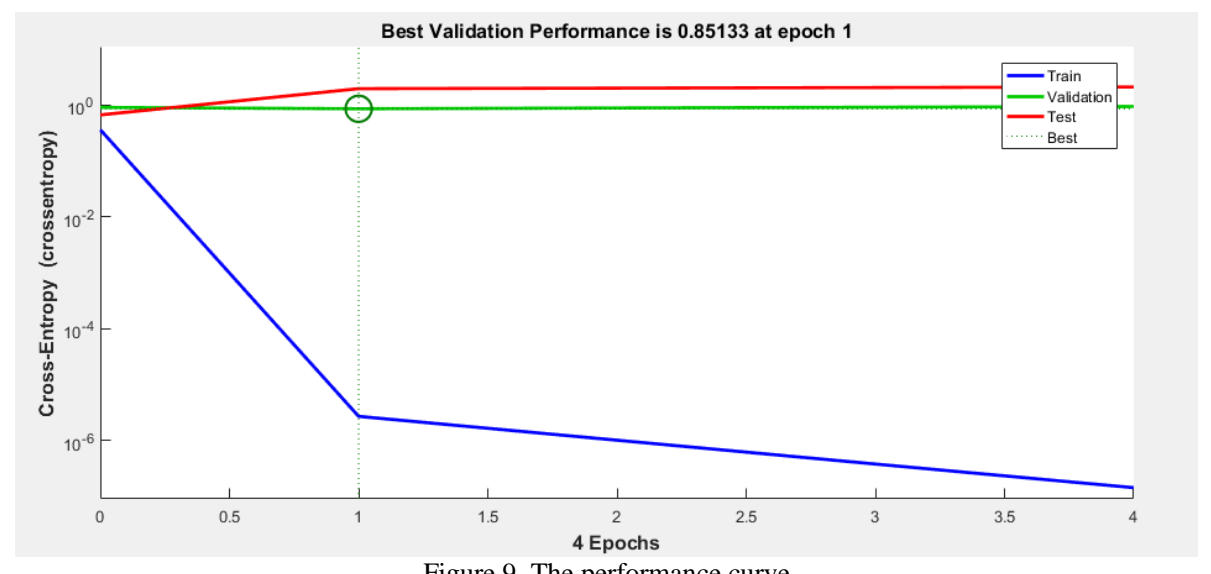

The training state curve is obtained in Figure 10.

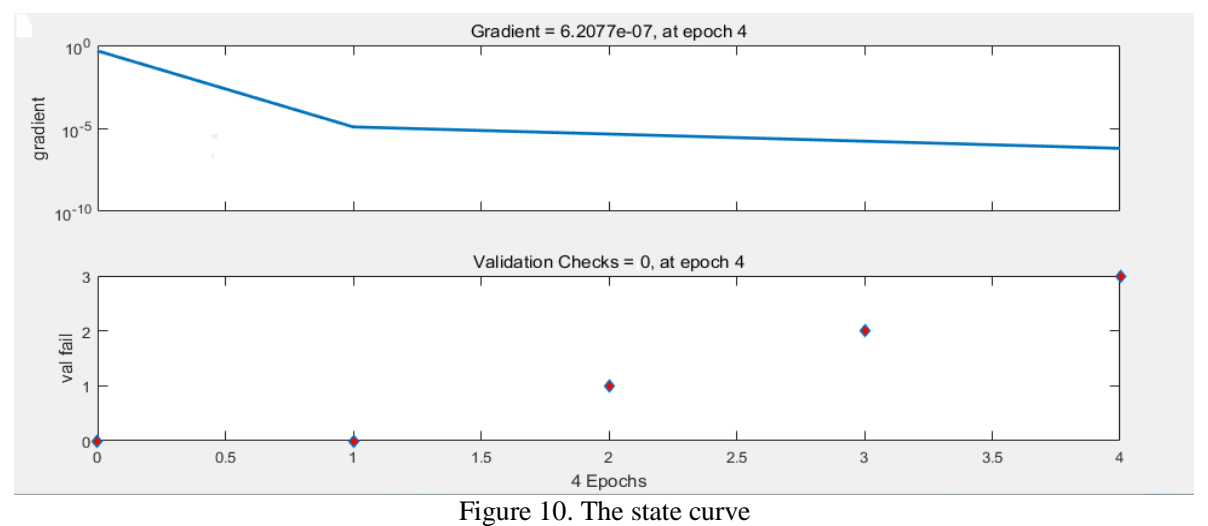

In the performance curve, the verification result at coordinate 1 is the best. It can be seen in Figure 9 that the training curve and the verification curve are completely inconsistent, which also indicates that the wind turbine blade has failed [25]. In the state curve, the descent of training gradient is relatively stable. The number of valid verification errors is 0 , and the verification results are relatively reliable and accurate. It is verified that the wind turbine blade with prefabricated cracks are faulty. This is very significant to the fault diagnosis of wind turbine blades.

\section{Conclusions}

In this paper, fatigue tests are carried out on a $1.5 \mathrm{~m}$ long NACA4418 airfoil blade of wind turbines, and the data of prefabricated cracked blade are obtained. On the LabVIEW software platform, the wavelet method is used to eliminate the noise of internal system and the noise introduced by the external environment. After the original data is filtered, the wavelet decomposition method is used to decompose the signal in four layers according to different frequencies of the signal. It can be seen that the $1.5 \mathrm{~m}$ long NACA4418 airfoil blade has a mutation peak at $0.14 \mathrm{~s}$ in the second layer decomposition signal and the fourth layer decomposition signal. The fault feather information of wind turbine blades is contained in the decomposition signals of different layers, which is helpful to identify faults of wind turbine blades. In order to further verify the reliability of the wavelet transform method for blade fault identification, the neural network is used to process the signal data. The 
reliability of the results in this paper is verified by the neural network training of different vibration frequency data. In this paper, the fault diagnosis technology is meaningful for blades, and the faults of wind turbine blades can be detected early on. This method is helpful to reduce the heavy losses caused by the failure of wind turbine blades. This paper provides a certain method for fault diagnosis of wind turbine blades, and it is helpful to the healthy operation of wind turbines.

\section{Acknowledgements}

This paper is supported by the Inner Mongolia Science \& Technology Plan Project (No. 102-510001), the Inner Mongolia Science and Technology Achievement Conversion Project (No. CHZH2018130), and the Inner Mongolia Autonomous Region Science \& Technology Innovation to Guide the Reward Project (No. 102-413128).

\section{References}

1. H. Badihi, Y. M. Zhang, and S. Rakheja, "Model-based Fault-Tolerant Pitch Control of an Offshore Wind Turbine," IFACPapers on Line, Vol. 51, No. 18, pp. 221-226, 2018

2. D. H. Wu and Y. J. Zhai, "Fault Diagnosis of Wind Turbine Pitch System based on System Identification Algorithms," Information and Control, Vol. 10, No. 5, pp. 563-574, 2016

3. Z. W. Gao and S. W. Sheng, "Real-Time Monitoring, Prognosis, and Resilient Control for Wind Turbine Systems," Renewable Energy, Vol. 116, No. 1, pp. 1-4, 2018

4. X. X. Zhang and Z. X. Liu, "Application of Resonance Demodulation and Wavelet Noise Reduction in Motor Fault Diagnosis," Electric Machines and Control, Vol. 2, No. 6, pp. 66-70, 2013

5. Z. H. Zhao, S. P. Yang, and Y. Q. Liu, "Application of Multi-Wavelet Coefficient Feature Extraction Method in Fault Diagnosis," Journal of Vibration, Measurement and Diagnosis, Vol. 12, No. 2, pp. 276-282, 2015

6. H. Z. Huang, X. D. Zhang, and D. B. Meng, "Multidisciplinary Design Optimization with Discrete and Continuous Variables of Various Uncertainties," International Journal of Computational Intelligence Systems, Vol. 5, No. 1, pp. 93-110, 2012

7. X. Yin, X. Y. Zhang, and X. Q. Chang, "Research on Early Fault Diagnosis Method of Wind Turbine based on AR-Hankel Matrix," Renewable Energy, Vol. 32, No. 1, pp. 80-85, 2016

8. T. Regan, C. Beale, and M. Inalpolat, "Wind Turbine Blade Damage Detection using Supervised Machine Learning Algorithms," Journal of Vibration and Acoustics, Vol. 139, No. 6, pp. 10-24, 2017

9. Y. G. Xu, Z. P. Meng, and M. Lu, "Application of Dual-Tree Complex Wavelet and Singular Difference Spectrum in Fault Diagnosis of Rolling Bearing," Journal of Vibration Engineering, Vol. 7, No. 6, pp. 965-973, 2013

10. L. J. Xu and L. Xu, "Test and Research on Static Load Test of $100 \mathrm{Kw}$ Wind Turbine Blade," Process Automation Instrumentation, Vol. 9, No. 7, pp. 37-42, 2016

11. M. A. Eder, F. Belloni, and A. Tesauro, "A Multi-Frequency Fatigue Testing Method for Wind Turbine Rotor Blades," Journal of Sound and Vibration, Vol. 388, pp. 123-140, 2017

12. S. X. Liu, L. P. Li, and T. Yu, "Diagnosis Technology of Wind Turbine Blade Icing State based on Vibration Detection," Proceedings of the CSEE, Vol. 9, No. 32, pp. 88-95, 2013

13. J. X. Bi, C. L. Zheng, and H. Z. Huang, "Load Analysis and Calculation Optimization of Horizontal Axis Wind Turbine Blades," International Journal of Performability Engineering, Vol. 14, No. 12, pp. 3098-3108, 2018

14. M. Gong and L. P. Li, "Summary of Research on Application of Acoustic Emission Technology in Wind Turbine Blade Fault Detection," Solar Energy, Vol. 1, No. 1, pp. 57-62, 2018

15. X. F. Chen, J. M. Li, and H. Cheng, "Research and Application of Condition Monitoring and Fault Diagnosis Technology in Wind Turbines," Journal of Mechanical Engineering, Vol. 47, No. 9, pp. 45-52, 2011

16. J. Z. Yan, L. Wang, and J. Zhou, "Development of on-Line Monitoring Terminal for Blade Cracking Defects of Wind Turbine," Electrical Automation, Vol. 3, No. 9, pp. 116-120, 2015

17. J. H. Zhang, J. Xiong, and M. F. Ren, "Filter-based Fault Diagnosis of Wind Energy Conversion Systems Subject to Sensor Faults," Journal of Dynamic Systems, Measurement, and Control, Vol. 138, No. 6, pp. 8-18, 2015

18. W. X. Yang, "Condition Monitoring the Drive Train of a Direct Drive Permanent Magnet Wind Turbine using Generator Electrical Signals," Journal of Vibration and Acoustics, Vol. 136, No. 2, pp. 21-28, 2015

19. B. J. Qiao, X. F. Chen, and X. J. Luo, "A Novel Method for Force Identification based on the Discrete Cosine Transform," Journal of Vibration and Acoustics, Vol. 137, No. 5, pp. 51-64, 2015

20. X. Wang, X. Pang, and Y. X. Wang, "Optimized VMD-Wavelet Packet Threshold Denoising based on Cross-Correlation Analysis," International Journal of Performability Engineering, Vol. 14, No. 9, pp. 2239-2247, 2018

21. L. Li, X. L. Zhang, and Y. H. Li, "Analysis of Coupled Vibration Characteristics of Wind Turbine Blade based on Green's Functions," Acta Mechanica Solida Sinica, Vol. 29, No. 6, pp. 620-630, 2015

22. B. Basu and A. Staino, "Control of a Linear Time-Varying System with a Forward Riccati Formulation in Wavelet Domain," Journal of Dynamic Systems, Measurement, and Control, Vol. 138, No. 10, pp. 72-78, 2016

23. M. Du, J. Yi, and J. B. Guo, "Application of Neural Network Technology in SCADA Data Analysis of Wind Turbines," Power System Technology, No. 7, pp. 2200-2205, 2018

24. Y. S. He, Y. Huang, and Z. M. Xu, "Fault Recognition of Motor Bearing based on Wavelet Singular Entropy and SOFM Neural Network," Journal of Vibration and Shock, Vol. 35, No. 10, pp. 217-223, 2017

25. P. L. Zhang, H. Chang, and J. Yang, "Application of BP Neural Network in AE Source Location of Cracks in Wind Turret Tube," China Measurement and Test, Vol. 12, No. 9, pp. 106-111, 2017 